\title{
Abundância e Diversidade de Coleoptera (Arthropoda: Insecta) de Solo em Fragmentos de Capoeira ao Entorno da Zona Urbana do Município de Uruará-PA, Brasil
}

\author{
Reinaldo Lucas Cajaiba ${ }^{\bowtie}$ \& Wully Barreto da Silva ${ }^{2}$
}

1. Universidade de Trás-os-Montes e Alto Douro, e-mail: reinaldocajaiba@hotmail.com (Autor para correspondência ${ }^{\varpi}$ ). 2. Universidade Federal do Pará, e-mail: wully_bio@hotmail.com.

\section{EntomoBrasilis 8 (1): 30-37 (2015)}

Resumo. A ação humana vem transformando as paisagens florestais em fragmentos isolados de remanescentes, podendo levar muitas espécies à extinção. Desta maneira se faz necessário a realização de estudos para o conhecimento e adequada caracterização da fauna de solo e, por conseguinte sua preservação. O objetivo do presente trabalho foi caracterizar a fauna de coleópteros em um fragmento florestal ao entorno da cidade de Uruará, PA. Para tanto, utilizou-se armadilhas tipo pitfall não iscadas para a coleta. O fragmento foi dividido em quatro transectos, com armadilhas instaladas ao longo da borda (T1), $50 \mathrm{~m}$ (T2), $100 \mathrm{~m}$ (T3) e $200 \mathrm{~m}$ (T4). Foram coletados 196 indivíduos, classificados em sete famílias e 34 espécies/morfoespécies. A família que apresentou a maior abundância foi a Curculionidae representando $56,40 \%$ da abundância, tendo o gênero Xyleborus sp. a maior dominância. Através do índice de Diversidade de Shanon, observou-se que a área de borda apresentou a menor diversidade e maior dominância (índice de Berger-Parker). Através da curva de acumulação e dos estimadores de espécies, ficou evidenciado que a área de estudo apresenta uma quantidade superior de espécies ao encontrado no presente estudo, e que pesquisas futuras são necessárias para um melhor acompanhamento das alterações que ocorrem nos fragmentos florestais com a finalidade de propor medidas de menor impacto e preservação dessa biodiversidade.

Palavras-Chave: Análise faunística; Biodiversidade; Coleoptera; Fragmento florestal; Insecta.

Abundance and Diversity of Ground Dwelling Beetles (Arthropoda: Insecta) in Fragments of Shrubbery Vegetation (Capoeira) in the Surroundings of the Urban Zone of Uruará City-PA, Brazil

Abstract. Human action has been transforming forest landscapes into isolated fragments, which may lead to the extinction of many species. Therefore, studies should be conducted to provide knowledge and the appropriate characterization of soil fauna, and, consequently, its preservation. This study was aimed to characterize the fauna of beetles in a forest fragment in the surroundings of Uruará city, PA. Unbaited pitfall traps were used for the collection of the beetles. The fragment was divided into four transects, with traps installed along the border (T1), $50 \mathrm{~m}(\mathrm{~T} 2), 100 \mathrm{~m}$ (T3) and 200 m (T4). 196 individuals classified in seven families and 34 species/morphospecies were collected. The most abundant family was the Curculionidae accounting for $56.40 \%$ of the abundance, with gender Xyleborus sp. being the most dominant. Measurement with the Shannon Diversity Index for measurement showed lower diversity and greater dominance (Berger-Parker index) in the border area. Using species accumulation curves and estimators it became evident that the study area had a greater number of species than those found in the present study, and that further studies are needed to better monitor changes in forest fragments in order to propose measures to reduce the impact on this biodiversity and preserve it.

Keywords: Beetles; Biodiversity; Faunal analysis; Forest fragment; Insecta.

lterações antrópicas têm causado, direta ou indiretamente, a redução e a perda de diversidade biológica (HALFFTER 2005; Verdú et al. 2007) devido à aceleração dos processos ecológicos naturais de extinção e surgimento de novas espécies, principalmente pela modificação, fragmentação e perda de habitat (MARQUES et al. 2002). As modificações na estrutura da vegetação influenciam diretamente a composição da fauna local (Doube \& Wardhalgh 1991; Oliveira 2001). Tal influência se dá pela mudança de características que afetam diretamente a biologia da espécie como: luminosidade, temperatura, umidade, que dão suporte para reprodução, nidificação, forrageamento e seu desenvolvimento (SCHWARZKopf \& RYLANDS 1989; MARINI 2001).

Os insetos têm recebido cada vez mais atenção como indicadores de ambientes terrestres, tanto por estarem presentes mesmo em áreas pequenas e fragmentadas, onde os vertebrados maiores já não existem, quanto por serem especialistas em recursos específicos e responderem rapidamente às alterações ambientais (LEWINSOHN et al. 2005; BARLOW et al. 2007; GARDNER et al. 2008).

Coleoptera representa um dos grupos animais mais diversos de vida na terra (LARA 1992; LAWRENCE et al. 1999). Existe cerca de 387.000 espécies de besouros descritas (Zhang 2011), o que corresponde a $40 \%$ das espécies de insetos conhecidas (GulLan \& CRANSTON 2005) e 25\% de todas as espécies animais e vegetais já descritas (RESH \& CARDÉ 2003). Os coleópteros ocupam virtualmente todos os ambientes, embora a grande maioria seja terrestre (GILLOT 2005).

Esse trabalho teve como objetivo conhecer a abundância e diversidade de coleópteros terrestres em uma região de cobertura florística de capoeira fragmentada no município de Uruará-PA.

\section{MATERIAL E MÉTODOS}

Local de estudo. O município de Uruará, Pará está localizado ao longo da Rodovia Transamazônica, mais precisamente no $\mathrm{km} \mathrm{180}$, com as seguintes coordenadas geográficas $03^{\circ} 43^{\prime} 27^{\prime \prime} \mathrm{S}$, $53^{\circ} 44^{\prime} \mathrm{O} 8^{\prime}$ ' W, no trecho entre os municípios de Altamira e Itaituba (Figura 1). As estações climáticas do ano na região apresentam um caráter bimodal de precipitação, uma época seca ou estiagem e outra chuvosa. O clima local, segundo a classificação de Köppen é do tipo Awi, típico de clima quente úmido, com média do total pluviométrico anual da ordem de $2000 \mathrm{~mm}$, cujo período mais chuvoso estende-se de dezembro a maio, e o menos chuvoso de junho a novembro (PEEL et al. 2007). A temperatura média anual 
T varia de 25 a $28{ }^{\circ} \mathrm{C}$ e umidade relativa do ar fica acima de $80 \%$ (CAJAiba 2014).

Coletas. As coletas foram realizadas no mês de novembro/2013, através de armadilhas de solo pitfall sem atrativos.

A área de coleta foi dividida em quatro transectos paralelos: Transecto 1 (T1) armadilhas instaladas entre a vegetação capoeira e pastagens; Transeco 2 ( T2) distando $50 \mathrm{~m}$ da área de borda; Transecto 3 ( $\mathrm{T}_{3}$ ) distando $100 \mathrm{~m}$ da área de borda; e Transcecto 4 (T4) distando 200m da área de borda. Em todos os transectos foram instalados quatro armadilhas distando $50 \mathrm{~m}$ uma da outra. As armadilhas permaneceram em campo por 36 horas. Como líquido conservante foi utilizado uma mistura de água, álcool e formol, além de algumas gotas de detergente neutro para quebrar a tensão superficial da água.

Análise estatística. Foi calculada a riqueza total e por transectos (S) e abundância total e por transectos (N). A diversidade do local estudado foi calculada através dos índices de Shannon-Wiener (H'). O índice de dominância de Berger-Parker (d) foi calculado para verificar o grau de dominância dos coleópteros (MAGURRAN 1988). A equitabilidade foi calculada através do índice de Pielou (J) (MagurRan 1988), através do software DivEs - Diversidade de Espécies 2.0 (RoDRIGUEs 2005). A riqueza estimada foi obtida através dos estimadores Jackknife 1 e 2, Chao 2 e Bootstrap. A análise de agrupamento das espécies presentes nos quatro pontos foram comparadas através da análise de agrupamento quantitativa para verificar se as espécies estão agrupadas em função do tipo de habitat (ZAR 1999), utilizando a distância euclidiana e realizado com auxílio do programa Systat 8.o.

O teste do Qui-quadrado $\left(\chi^{2}\right)$ foi aplicado para verificar se existe alguma diferença de abundância entre os transectos e o grupo estudado. Calculamos as curvas esperadas de acumulação de espécies (curvas de rarefação baseadas em amostras, segundo Gotelli \& COLWELL 2001) com os respectivos intervalos de confiança a 95\% de probabilidade, utilizando as fórmulas analíticas apresentadas em CoLwell et al. (2004), através do software PAST 1.75 (HAMmER et al. 2001).

\section{RESULTADOS E DISCUSSÃO}

Foram coletados 196 indivíduos distribuídos em sete famílias e 34 espécies/morfoespécies. A espécie que apresentou a maior abundância foi a Xyleborus sp1 e Xyleborus affinis Eichhoff, com 63 espécimes e 26 espécimes, respectivamente, estando ausente apenas no T4. A família que apresentou a maior dominância nos transectos T1, T2 e T3 foi a Curculionidae, subfamília Scolytinae, sendo responsável por $56,40 \%$ da abundância total dos espécimes coletados, sendo a mesma que apresentou a maior abundância (110 espécimes) (Tabela 1$)$.

Em estudos realizados por PeLENTIR (2007) em florestas do MT,

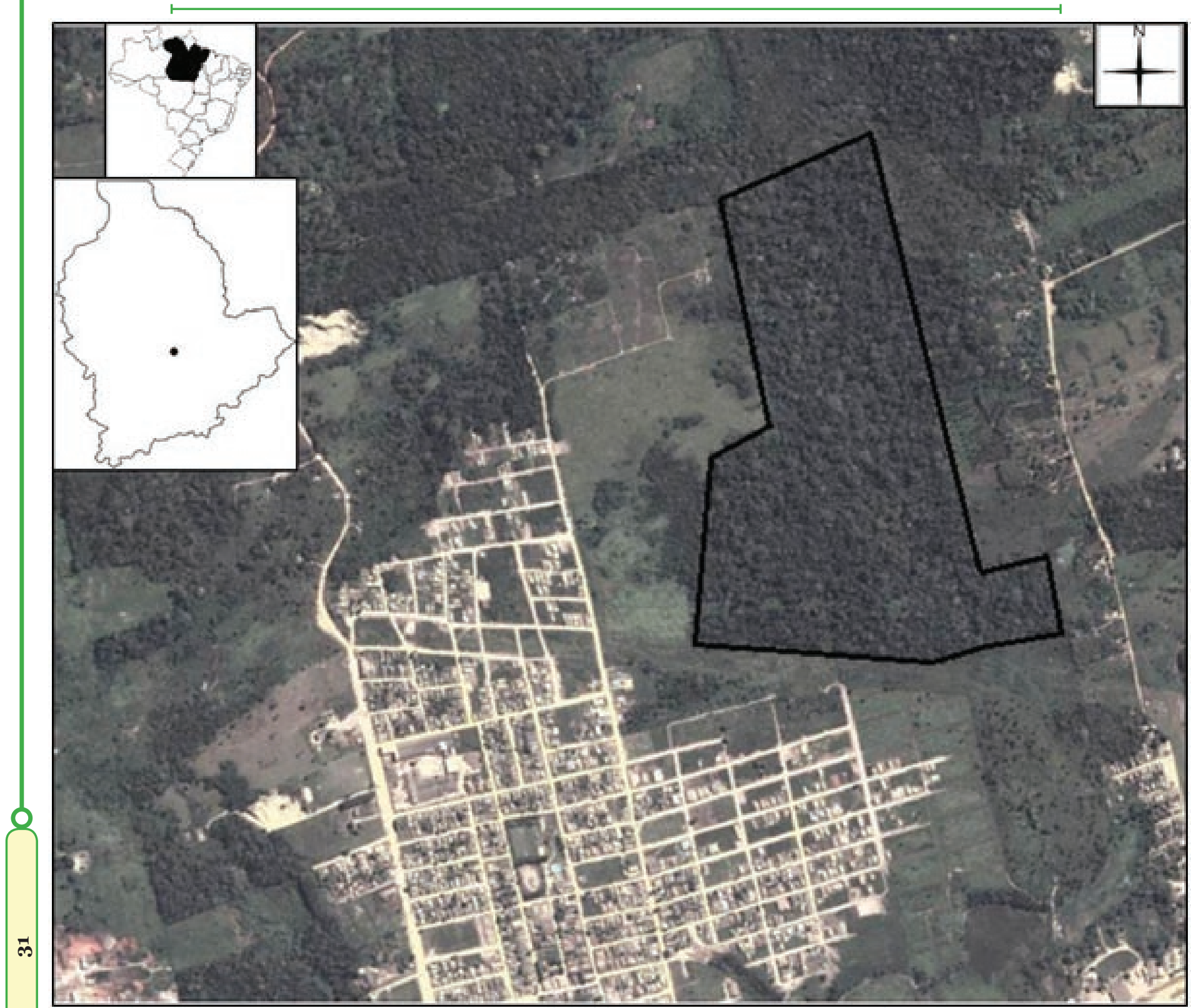

Figura 1. Localização da área de estudo no município de Uruará, PA. Fonte: Google Earth, 2013. 
Dorval \& Peres-Filho (2001) e Dorval et al. (2004) em trabalhos desenvolvidos também em MT, em fragmentos de cerrado e em talhões de Eucalyptus spp., coletaram uma maior quantidade de espécies de escolitídeos pertencente à Xyleborini, que são espécies tropicais, geralmente responsáveis pela degradação de galhos e pequenos ramos lenhosos, no entanto, são espécies capazes de produzir surtos populacionais em talhões cuja fitossanidade esteja comprometida. Estas espécies são consideradas pantropicais, ou seja, estão presentes em todas as regiões tropicais (RocHA 2010).

Sistemas mais simplificados podem proporcionar vantagens a determinadas espécies que terão sua população elevada ultrapassando populações em sistemas naturais (DAvis et al. 2001; Escobar et al. 2008), e estes alteram a abundância total.

A grande abundância dessas espécies nessa área de estudo pode estar relacionada à grande quantidade de material com restos vegetais, tocos e galhos quebrados, que podem servir como local de desenvolvimento e fornecer condições para o crescimento populacional dessas espécies (PEREIRA 2006).

Em relação ao número de famílias o T1 foi onde verificou-se a menor quantidade (cinco famílias), os demais transectos apresentaram seis cada. Este baixo grau de diversidade das famílias pode estar relacionado ao intenso uso do solo no passado,

Tabela 1. Abundância (N) e Riqueza (S) de coleópteros coletados em quatro transectos ao longo de um fragmento florestal de capoeira localizada no município de Uruará, PA. T1 (Transecto 1), T2 (Transecto 2), T3 (Transecto 3) e T4 (Transecto 4), no mês de Novembro/2013.

\begin{tabular}{|c|c|c|c|c|c|}
\hline Família/Espécie/Mofoespécie & T1 & T2 & T3 & T4 & Total \\
\hline \multicolumn{6}{|l|}{ Carabidae } \\
\hline Clivina sp. & 1 & o & 1 & o & 2 \\
\hline Polpochila sp. & 2 & 1 & o & o & 3 \\
\hline Lebiini sp. & 1 & 2 & o & o & 3 \\
\hline Carabidae 3 & o & o & 1 & 1 & 2 \\
\hline Carabidae 4 & o & 1 & 1 & o & 2 \\
\hline \multicolumn{6}{|l|}{ Histeridae } \\
\hline Euspilotus sp1 & o & o & 3 & 1 & 4 \\
\hline \multicolumn{6}{|l|}{ Scarabaeidae } \\
\hline Ateuchus sp. & o & 2 & o & 2 & 4 \\
\hline Canthidium sp. & o & 3 & 4 & 2 & 9 \\
\hline Canthidium sp. & o & 1 & 1 & o & 1 \\
\hline Deltochilum sp. & o & 1 & 4 & o & 5 \\
\hline Dichotomius sp. & o & o & 1 & 1 & 2 \\
\hline Pseudocanthon aff. xanthurus Blanchard & o & o & o & 2 & 2 \\
\hline Ontherus pubens Génier & o & o & o & 1 & 1 \\
\hline \multicolumn{6}{|l|}{ Curculionidae } \\
\hline \multicolumn{6}{|l|}{ Staphylinidae } \\
\hline Belonuchus sp2. & 1 & 1 & o & o & 2 \\
\hline Xantholinus sp. & o & 1 & 1 & 0 & 2 \\
\hline Staphylinidae 1 & 1 & 1 & 2 & o & 4 \\
\hline Staphylinidae 2 & 3 & 1 & 1 & 0 & 5 \\
\hline Staphylinidae 3 & 1 & 1 & o & o & 2 \\
\hline Staphylinidae 4 & 1 & 1 & o & 0 & 1 \\
\hline \multicolumn{6}{|l|}{ Não identificado } \\
\hline NI 1. & 1 & o & o & 1 & 2 \\
\hline NI 2. & 1 & 1 & 0 & o & 2 \\
\hline $\mathrm{NI} 3$. & 0 & o & 1 & 1 & 2 \\
\hline Riqueza (S) & 19 & 21 & 20 & 10 & - \\
\hline Abundância (N) & 73 & 46 & 64 & 13 & 196 \\
\hline
\end{tabular}


juntamente com o fato de ser um fragmento isolado por uma matriz altamente urbanizada, a qual prejudica os processos de sucessão e recolonização (BEIROZ et al. 2010). Quando analisou-se a riqueza de espécies, o transecto que apresentou a menor riqueza foi o T4 com dez espécies, seguido por T1, T3 e T2 com 19, 20 e 21 espécies, respectivamente. $\mathrm{O}$ transecto que verificou-se a maior abundância foi o T1 com 73 espécimes, seguido pelo T3 com 64 espécimes, sendo o T4 o menos abundante (13 espécimes).

Nenhuma espécie foi comum a todos os transectos. As espécies Pseudocanthon aff. xanthurus Blanchard e Ontherus pubens Génier, ambas das famílias Scarabaeidae foram encontradas exclusivamente no $\mathrm{T}_{4}$ (Tabela 1 ).

O gênero Pseudocanthon possui três espécies continentais nas Américas (as demais são antilhanas) (MATTHEws 1966). $P$. aff. xanthurus, a única espécie registrada neste trabalho, já foi coletada em áreas de savana intra-amazônica (Matavelli \& LouZADA 2008) e em ambientes abertos como pastagens (SCHIFlLer 2003), e é relatada em 15 Estados brasileiros (LouzADA et al. 2007). Muito provavelmente $P$. aff. xanthurus consiste num complexo de espécies de difícil identificação, já que sua distribuição é descontínua (KorASAKI et al. 2012), neste mesmo estudo, no município de Benjamin Constante, AM, estes autores encontraram essa espécie como uma das mais abundantes, porém, utilizando armadilhas pitfall iscadas.

O gênero Ontherus, conta com três subgêneros e algumas dezenas de espécies nas Américas do Sul e Central. As duas espécies capturadas pertencem ao subgênero nominotípico. $O$. pubens é distribuída por toda a bacia amazônica e tem hábitos copronecrófagos segundo estudo realizado por VAZ-DE-MELLo (1999), onde no estado do Acre foi encontrada apenas quatro espécimes, porém, utilizando armadilhas iscadas e interceptação em voô. As espécies deste gênero são em geral coprófagas, e um grupo de espécies é obrigatoriamente associado a detritos acumulados em ninhos de formigas dos gêneros Atta e Acromyrmex. Este gênero já foi relatado em abóboras (Curcubita sp.) podres e voando em volta de lâmpadas; algumas espécies vivem em colônias de 20 a 30 indivíduos em ninhos das formigas do gênero Acromyrmex (LUEDERWALDT 1931). Esta espécie por ter sido coletada apenas no $\mathrm{T} 4$, transecto mais distante da borda (Tabela 1), pode ser uma espécie ocasional de ambiente mais fechado (VAZ-DE-MELLO 1999; KORASAKI et al. 2012).

$\mathrm{O}$ índice de Shanon foi maior no T2 $\left(\mathrm{H}^{\prime}=2,51\right)$ seguido pelo T3 $\left(H^{\prime}=2,45\right)$, tendo a menor diversidade o transecto T1 com H' = 2,09. O índice de dominância de Berger-Parker mostrou que o transecto que apresentou a maior dominância foi o T1 com 0,43 e o T4 apresentando a menor dominância $(d=0,18)$. Essa alta dominância no T1 é devido à grande abundância das espécies Xyleborus sp. O índice de Equitabilidade de Pielou que mede a proporção da diversidade observada com relação à máxima diversidade esperada (MAGURRAN 1988) mostra que o transecto $\mathrm{T} 4$ apresentou o maior índice $(\mathrm{J}=0,97)$, enquanto o transecto T1 apresentou o menor índice $(\mathrm{J}=0,71)$ (Tabela 2). A baixa equidade no T1 em relação aos demais transectos, reflete a alta dominância de Xyleborus sp. na amostra. Locais com fatores limitantes atuando intensamente e competição interespecífica, costumam apresentar baixos índices de diversidade, aumentam o número de espécies mais comuns e diminuem as raras, tornando o local mais específico (Silveira-Neto et al. 1976).

Apesar de ser bastante antroponizada, pela expansão da zona urbana e por atividades agropecuárias, a área estudada apresenta diversidade relativamente alta, que segundo THомаZINI \& Thomazini (2000) as florestas secundárias também podem abrigar grande diversidade de insetos, sendo que a variação na idade e tamanho das mesmas pode influenciar essa diversidade.

A análise quantitativa (Distância Euclidiana) agrupou com maior similaridade os transectos $\mathrm{T} 1 \mathrm{e} \mathrm{T} 2$ devido à maior similaridade das espécies coletadas nesses locais, sendo o transecto $\mathrm{T}_{4} \mathrm{o}$ mais distante dos demais (Figura 2). Através do teste do Quiquadrado, observamos diferença significativa entre T1 e T3 $\left(\chi^{2}=\right.$ 46,67; $\mathrm{p}<0,05)$ e entre T2 e T4 $\left(\chi^{2}=46,16 ; \mathrm{p}=0,01\right)$.

Tabela 2. Diversidade de Shannon-Wienner (H'); Equitabilidade de Pielou (J') e Dominância de Berger-Parker (d) para os quatro transectos estudados no fragmento florestal no município de Uruará, PA, no mês de Novembro/2013.

\begin{tabular}{lcccc}
\hline \multicolumn{1}{c}{ Índices } & T1 & T2 & T3 & T4 \\
\hline$H^{\prime}$ & 2,09 & 2,51 & 2,45 & 2,24 \\
\hline J & 0,71 & 0,82 & 0,81 & 0,97 \\
D & 0,43 & 0,34 & 0,23 & 0,18 \\
\hline
\end{tabular}

Diversos estudos citam diferenças entreáreas de borda, ambientes com diferentes níveis de fragmentação ou cobertura vegetal (SPector \& AyZAMa 2003; Pohl et al. 2007; GARDNer et al. 2008; Sовек et al. 2009), nos quais a maior cobertura ou complexidade sustentam comunidades mais estáveis e/ou diversas (EISENHAUER et al. 2008).

O isolamento dos fragmentos urbanos limita a chegada de coleópteros de outras áreas, aumentando o risco de extinção de espécies. Dessa forma, o isolamento inibe o efeito resgate que poderia evitar essas extinções (BROwN \& KoDRIK-BROwN 1977; Cielo-Filho \& SANTin 2002) e a competição com espécies introduzidas, podendo levar à extinção de espécies nativas e diminuindo as interações mutualísticas que poderiam favorecer um aumento da biodiversidade (JANZEN 1983; DÁTTILO et al. 2009; Cajaiba \& Silva 2014). Por outro lado, vale considerar que todos os ambientes estudados estão inseridos no mesmo fragmento urbano e sujeitos a um alto grau de isolamento e recolonização. Logo, a similaridade entre os ambientes possivelmente indica que todo o fragmento tenha sido alterado pelos efeitos de borda e pela constante ação antrópica no local (DÁtTiLo et al. 2009; CAJAIBA \& Silva 2014).

A curva de acumulação de espécies em função do esforço amostral, de acordo com a sequência de amostragem adotada, apresenta uma evolução que não estabiliza, sugerindo que o número de espécies de coleópteros existente na região de estudo, seja superior às identificadas no presente trabalho (Figura 3). Deste modo, a riqueza observada no presente estudo deve ser tomada como uma subestimativa, decorrente do esforço amostral, pois segundo Melo et al. (2003), a riqueza observada de espécies é fortemente correlacionada ao esforço de amostragem. De fato, a estimativa efetuada da riqueza potencial de espécies de coleópteros existentes, na região, variou entre 33 e 41, dependendo do método utilizado (Figura 4).

Apesar de o local estudado ter sofrido grande fragmentação nos últimos anos devido ao crescimento da cidade em direção ao seu entorno e às atividades pecuárias, a região ainda apresenta uma rica fauna. Como não existem estudos anteriores aos episódios de fragmentação não é possível fazer uma comparação dos efeitos da fragmentação neste local. É necessário, portanto, maior atenção para o estudo de paisagens modificadas pelo homem, situações cada vez mais comuns atualmente (MORENO \& HALFFTER 2001; FERRAZ et al. 2009).

Por se tratar de uma área verde, urbana e sujeita a ação antrópica é importante a realização de estudos posteriores com outros grupos de artrópodes sensíveis as alterações ambientais (OLIVER \& BEATTIE 1996; DÁtıllo et al. 2011) a fim de acompanhar suas populações visando a conservação desse importante remanescente florestal da cidade de Uruará, PA.

As análises dos dados sobre os coleópteros neste fragmento de estudo que habitam o solo indicam que: a abundância dos 


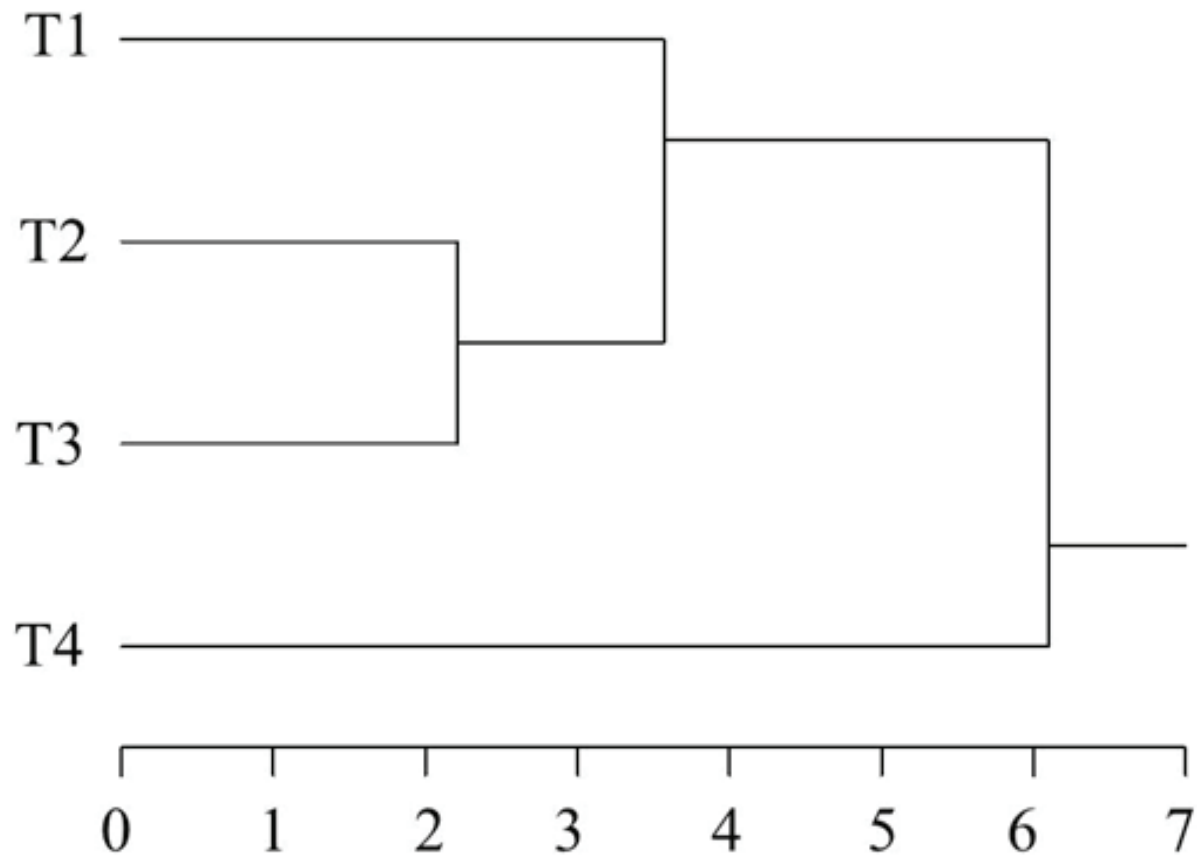

Figura 2. Dendograma de análise quantitativa (Distância Euclidiana) com agrupamento de similaridade entre os transectos estudados, no mês de Novembro/2013.
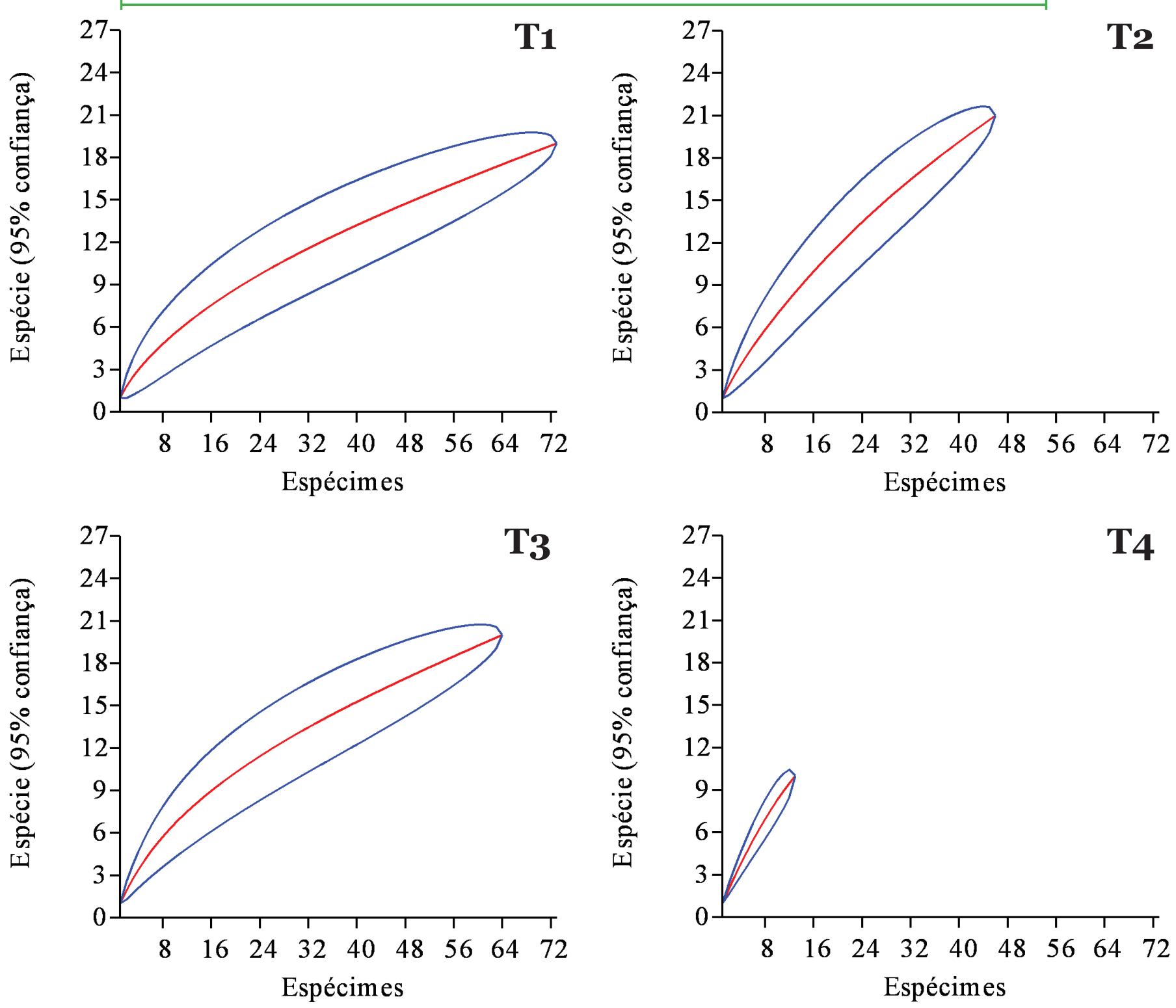

Figura 3. Curva de acumulação de espécies (linha central - em vermelho) e intervalos de confiança de 95\% (linhas externas - em azul) para os quatro transectos, no mês de Novembro/2013. 

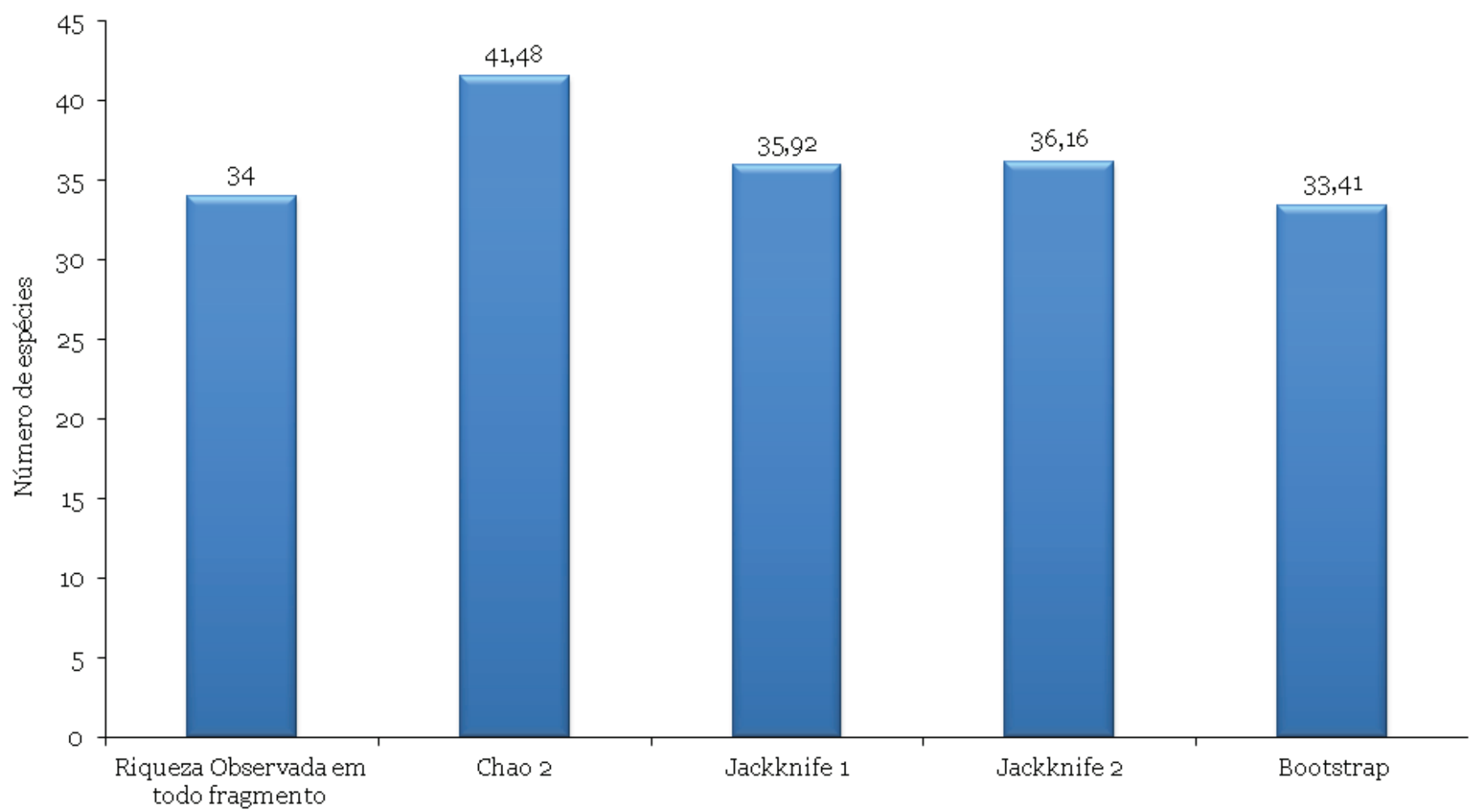

Estimadores

Figura 4. Riqueza observada e estimada de coleópteros para os quatro transectos no fragmento florestal no município de Uruará, PA, no mês de Novembro/2013.

coleópteros do solo na borda é muito superior à da área do interior, porém, com uma menor diversidade e maior dominância; pelo fato de nem todas as espécies de coleópteros que ocorrem no fragmento terem sido amostradas, como também indicado pelas curvas de rarefação de espécies, estudos como este mostram a importância da realização de inventários de longo prazo e da associação de diferentes métodos de amostragem; apesar de o local estudado ser bastante fragmentado e reduzido, os resultados reforçam a necessidade de conservação dos remanescentes florestais, que apesar de abrigar grande diversidade estão ameaçados pelos efeitos da fragmentação florestal.

\section{REFERÊNCIAS}

Barlow, J., T.A. Gardner, I.S. Araujo, T.C. Avila-Pires, A.B. Bonaldo, J.E. Costa, M.C. Esposito, L.V. Ferreira, J. Hawes, M.I.M. Hernández, M.S. Hoogmoed, R.N. Leite, N.F. LoMan-Hung, J.R. Malcolm, M.B. Martins, L.A.M. Mestre, R. Miranda-Santos, A.L. Nunes-Gutjahr, W.L. Overal, L. Parry, S.L. Peters, M.A. Ribeiro-Junior, M.N.F. Silva, C. Silva Motta \& C.A. Peres, 2007. Quantifying the biodiversity value of tropical primary, secondary, and plantation forests. Proceedings of the National Academy of Sciences of the United States of America, 104: 18555-18560.

Beiroz, W., A.S. Zaú \& E. Castro Jr, 2010. Impacto das Estradas na Distribuição de Besouros em um Fragmento de Mata Atlântica de Encosta no Parque Nacional da Tijuca, Rio de Janeiro, RJ. EntomoBrasilis, 3: 64-68.

Brown, J.H. \& A. Kodric-Brown, 1977. Turnover rates in insular biogeography: effect of immigration on extinction. Ecology, 58: 445-449.

Cajaiba, R.L. \& W.B. Silva. 2014. Mirmecofauna (Hymenoptera, Formicidae) em fragmento florestal urbano no município de Uruará-PA. Enciclopédia Biosfera, 18: 2226-2238.

Cajaiba, R.L., 2014. Morcegos (Mammalia, Chiroptera) em cavernas no município de Uruará, Pará, norte do Brasil. Biota Amazônica, 4: 81-86.

Cielo-Filho, R. \& D.A. Santin, 2002. Estudo florístico e fitossociológico de um fragmento florestal urbano - Bosque dos Alemães, Campinas, SP. Revista Brasileira de Botânica,
25: 291-301.

Colwell, R.K., C.X. Mao \& J. Chang, 2004. Interpolatin, extrapolatin, and comparing incidence-based species accumulation curves. Ecology, 85: 2717-27.

Dáttilo, W., E.C. Marques, J.C.F. Falcão \& D.D.O. Moreira, 2009. Interações mutualísticas entre formigas e plantas. EntomoBrasilis, 2: 32-36.

Dáttilo, W., N. Sibinel, J.C.F. Falcão \& R.V. Nunes, 2011. Mirmecofauna em um fragmento de floresta atlântica urbana no município de Marília, SP, Brasil. Biocience Journal, 27: 494-504.

Davis, A.J., J.D. Holloway, H. Hujbregts, J. Krikken, A.H. KirkiSpriggs \& S.L. Sutton, 2001. Dung beetles as indicators of change in the forests of northern Borneo. Journal of Applied Ecology, 38: 593-616.

Dorval, A. \& O. Peres-Filho, 2001. Levantamento e flutuação populacional de coleópteros em vegetação do cerrado da baixada cuiabana, MT. Ciência Florestal, 11: 171-182.

Dorval, A., O. Peres-Filho \& E.N. Marques, 2004. Levantamento de Scolytidae (Coleoptera) em plantações de Eucalyptus spp. em Cuiabá, MT. Ciência Florestal, 14: 47-58.

Doube, B.M. \& K.G. Wardhalgh. 1991. Habitat associations and niche parttioning in an island dung beetle community. Acta Oecologica, 12: 451-459.

Eisenhauer, N., A. Milcu, C.W. Sabais \& S. Scheu, 2008. Animal Ecosystem Engineers Modulate the Diversity-Invasibility Relationship. Public Library of Science ONE, 3: e3489. doi:10.1371/journal.pone.0003489.

Escobar, F., G. Halffter, A. Solís, V. Halffter, \& D. Navarrete, 2008. Temporal shifts in dung beetle community structure within a protected area of tropical we forest: a 35-year study and its implictions for long-term conservation. Journal of Applied Ecology, 45: 1584-1592.

Ferraz, A., P. Cristina, B.A. Gadelha \& V.M. Aguiar-Coelho, 2009. Análise faunística de Calliphoridae (Diptera) da Reserva Biológica do Tinguá, Nova Iguaçu, Rio de Janeiro. Revista Brasileira de Entomologia, 53: 620-628.

Gardner, T. M.I.M. Hernández, J. Barlow \& C.A. Peres, 2008. Understanding the biodiversity consequences of habitat 
change: the value of secondary and plantation forests for neotropical dung beetles. Journal of Applied Ecology, 45:111.

Gillott, C., 2005. Entomology, $3^{\text {rd }}$ ed. Dordrecht: Springer, 831p. Gotelli, N.J. \& R.K. Colwell, 2001. Quantifying biodiversity: procedures and pitfalls in the measurement and comparison of species richness. Ecology Letters, 4: 379-391.

Gullan, P.J. \& P.S. Cranston, 2005. The insects - An outline of entomology, 3 ed. Carlton: Blackwell Publishing, 505p.

Halffter, G. \& E.G. Matthews, 1966. The natural history of dung beetles of the subfamily Scarabaeinae (Coleoptera: Scarabaeidae). Folia Entomológica Mexicana, 12: 1-312.

Hammer, O., D.A.T. Harper \& P.D. Ryan, 2001. PAST: Paleontological Statistics software package for education data analysis. Paleontologia electronica, 4: 9p.

Janzen, D.H., 1983. No parks is an island: increasing interference from outside as park size decreases. Oikos, 41: 402-410.

Korasaki, V., F.Z. Vaz-de-Mello, R.F. Braga, R. Zanetti \& J. Louzada, 2012. Taxocenose de Scarabaeinae (Coleoptera: Scarabaeidae) em Benjamin Constant, AM. Acta Amazonica, 42: 423-432.

Lara, F.M., 1992. Princípios de Entomologia. São Paulo: Ícone, 331p.

Lawrence, F.A., A.M. Hasting, M.J. Dallwitz, T.A. Paine \& E.F. Zurcher, 1999. Beetles of the world. A key and information system for families and subfamilies (Version 1.0 for MSWindows, CD-ROM). CSIRO Publishing. Melbourne, Australia.

Lewinsohn, T.M., A.V.L. Freitas \& P.I. Prado, 2005. Conservation of terrestrial invertebrates and their habitats in Brazil. Conservation Biology, 19: 640-645.

Louzada, J.N.C., F.S. Lopes \& F.Z. Vaz-de-Mello, 2007. Structure and composition of a dung beetle community (Coleoptera, Scarabaeinae) in a small forest patch from Brazilian Pantanal. Revista Brasileira de Zoociências, 9: 199-203.

Luederwaldt, H., 1931. The genus Ontherus (Coleop.) (LamellicCoprid.-Pinot) with a key to determining the Americans Pinotides. Revista do Museu Paulista, 17: 363-423.

Magurran, A.E, 1988. Ecological diversity and its measurement. Princeton, Princeton University Press, 179p.

Marini, M., 2001. Effects of fragmentation on birds of the cerrado region, Brazil. Bird Conservation International, 11: 11-23.

Marques, A.A.B., C.S. Fontana, E. Vélez, G.A. Bencke, M.Schneider \& R.E. Reis, 2002. Lista de Referência da Fauna Ameaçada de Extinção no Rio Grande do Sul. Decreto no 41.672, de 10 de junho de 2002. Porto Alegre: FZB/MCTPUCRS/ PANGEA, $52 \mathrm{p}$.

Matavelli, R.A. \& J.N.C. Louzada, 2008. Invasão de áreas de savana intra-amazônicas por Digitonthophagus gazella (Fabricius, 1787) (Insecta: Coleoptera: Scarabaeidae). Acta Amazonica, 38:153-158.

Matthews, E.G. 1966. A taxonomic and zoogeographic survey of the Scarabaeinae of the Antilles (Coleoptera: Scarabaeidae). Memoirs of the American Entomological Society, 21: 1-134.

Melo, A.S., R.A.S. Pereira, A.J. Santos, G.J. Shepherd, G. Machado, H.F. Medeiros \& R.J. Sawaya, 2003. Comparing species richness among assemblages using sample units: Why not use extrapolation methods to standardize different sample sizes? Oikos, 101: 398-410.

Moreno, C.E. \& G. Halffter, 2001. Spatial and temporal analysis of $\mathrm{a}, \mathrm{b}$ and $\mathrm{g}$ diversities of bats in a fragmented landscape. Biodiversity and Conservation, 10: 367-382.

Oliveira, M.L., 2001. Stingless bee and orchid bees (Euglossini) in Terra Firme tropical forest and forest fragments, p. 208-219. In: Bierregaard, R.O., C. Gascon,T.E. Lovejoy, R. Mesquita (Eds). Lessons from Amazonia - the ecology and conservation of a fragmented forest. Michigan: Sheridan Books, 219p.

Oliver, I. \& A.J. Beattie, 1996. Designing a cost-effective invertebrate survey: a test of methods for rapid assessment of biodiversity. Ecology Applied, 6: 594-607.
Peel, M.C., B.L. Finlayson \& T.A. McMahon, 2007, Updated world map of the Köppen-Geiger climate classification. Hydrology and Earth System Sciences, 11: 1633-1644, doi:10.5194/hess11-1633-2007.

Pelentir, S.C.S., 2007. Eficiência de cinco modelos de armadilhas etanólicas na coleta de Coleoptera: Scolytidae, em floresta nativa no município de Itaara, RS. Dissertação (Mestrado em Engenharia Florestal), Universidade Federal de Santa Maria. $74 \mathrm{p}$.

Pereira, R.A. 2006. Scolytidae em povoament em Pinus spp. em Telêmaco Borba, PR. Dissertação (Mestrado em Ciências Biológicas), Universidade Federal do Paraná. 51 p.

Pohl, G.R., D.W. Langor \& J.R. Spence, 2007. Rove beetles and ground beetles (Coleoptera: Staphylinidae, Carabidae) as indicators of harvest and regeneration practices in western Canadian foothills forests. Biological Conservation, 137: 294307.

Resh, V.H. \& R.T. Cardé (eds.), 2003. Encyclopedia of Insects. Orlando: Academic Press, 1266 p.

Rocha, J.R.M., 2010. Ocorrência e dinâmica populacional de Scolytidae, Bostrichidae e Platypodidae em povoamentos de eucaliptos e fragmento de cerrado, no município de Cuiabá-MT. Dissertação (Mestrado em Ciências Florestais e Ambientais) - Universidade Federal de Mato Grosso. 63 p.

Rodrigues, W.C., 2005. DivEs - Diversidade de espécies. Versão 2.0. Software e Guia do Usuário, 2005. Disponível em: <http://ww.ebras.bio.br/dives >. [Acesso em 22.11.2013].

Schiffler, G., 2003. Fatores determinantes da riqueza local de espécies de Scarabaeidae (Insecta: Coleoptera) em fragmentos de Floresta Estacional Semidecídua. Dissertação (Mestrado), Universidade Federal de Lavras. 68p.

Schwarzkopf, L. \& A.B. Rylands, 1989. Primate species richness in relation to habitat structure in Amazonian rainforest fragments. Biological Conservation, 48: 1-12.

Silveira-Neto, S., O. Nakano, D. Barbin \& N.A.V. Nova, 1976. Manual de ecologia de insetos. São Paulo, Ed. Agronômica Ceres, 419p.

Sobek, S., I. Steffan-Dewenter, C. Scherber \& T. Tscharntke, 2009. Spatiotemporal changes of beetle communities across a tree diversity gradient. Diversity and Distributions, 15: 660670.

Spector, S. \& S. Ayzama, 2003. Rapid Turnover and Edge Effects in Dung Beetle Assemblages (Scarabaeidae) at a Bolivian Neotropical Forest-Savanna Ecotone. Biotropica, 35: 394404.

Thomazini, M.J. \& A.P.B.W. Thomazini, 2000. A fragmentação florestal e a diversidade de insetos nas florestas tropicais úmidas. Rio Branco: Embrapa Acre. 21 p. Disponível em <http://www.cpafac.embrapa.br> Acesso em 20.11.2013.

Vaz-de-Mello, F.Z., 1999. Scarabaeidae s. str. (Coleoptera: Scarabaeoidea) de um Fragmento de Floresta Amazônica no Estado do Acre, Brasil. Anais da Sociedade Entomológica do Brasil, 28: 447-453.

Verdú, J. R., C.E. Moreno, G. Sánchez-Rojas, C. Numa, E. Galante \& G. Halffter, 2007. Grazing promotes dung beetle diversity in the xeric landscape of a Mexican Biosphere Reserve. Biological Conservation, 140: 308-317.

Zar, J.H., 1999. Biostatitical Analysis. $3^{\text {rd }}$ ed. New Jersey, Prentice Hall.

Zhang, Z.Q., 2011. Phylum Arthropoda von Siebold, 1848 In: Zhang, Z.Q. (Ed.) Animal biodiversity: An outline of higherlevel classification and survey of taxonomic richness. Zootaxa, 4138: 99-103.

\section{Recebido em: 30/12/2013}

Aceito em: 30/10/2014 
Como citar este artigo:

Cajaiba, R.L. \& W.B. da Silva, 2015. Abundância e Diversidade de Coleópteros de Solo em Fragmentos de Capoeira ao Entorno da Zona Urbana do Município de Uruará-PA, Brasil. EntomoBrasilis, 8 (1): 30-37.

Acessível em: doi:10.12741/ebrasilis.v8i1.414
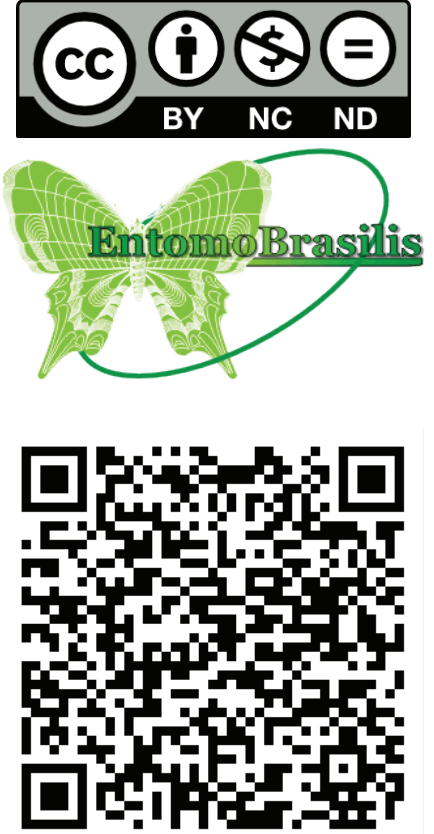